\title{
Complex spin configurations in hybrid magnetic multilayer structures due to mutual spin imprinting
}

\author{
Matthew T. Bryan, ${ }^{1}$ Georg Heldt, ${ }^{2,3}$ Thomas Thomson, ${ }^{2}$ Laura J. Heyderman, ${ }^{3,4}$ and Gino Hrkac ${ }^{1, *}$ \\ ${ }^{1}$ College of Engineering, Mathematics and Physical Sciences, University of Exeter, EX4 4QF, United Kingdom \\ ${ }^{2}$ School of Computer Science, University of Manchester, Oxford Road, Manchester, M13 9PL, United Kingdom \\ ${ }^{3}$ Laboratory for Micro- and Nanotechnology, Paul Scherrer Institute, 5232 Villigen PSI, Switzerland \\ ${ }^{4}$ Laboratory for Mesoscopic Systems, Department of Materials, ETH Zurich, 8093 Zurich, Switzerland \\ (Received 4 October 2013; revised manuscript received 5 August 2016; published 14 September 2016)
}

\begin{abstract}
Hybrid Co/Pd-Permalloy multilayer structures exhibit multiple domain phases resulting from mutual spin imprinting, dependent on the precise layer composition and thickness. While such hybrid structures can support either a pure Landau closure-domain pattern or a perpendicular exchange-spring magnetization structure, numerical analysis also revealed an anomalous mixed Landau-maze domain state. Mutual imprinting of the spin configuration between the layers was strongest for the latter multidomain state, which we propose is a consequence of exchange energy dissipation over the two lateral dimensions. An analytical derivation of domain phase boundaries was consistent with the micromagnetic simulations and gave important insight into their origin.
\end{abstract}

DOI: 10.1103/PhysRevB.94.104415

\section{INTRODUCTION}

When two or more materials are combined, the resulting hybrid structure may yield enhanced performance compared to the individual components [1]. Hybrid multilayer structures may exhibit novel magnetic properties, such as exchange bias, time-reversal asymmetry with respect to a changing applied field [2-4], or altered anisotropy symmetry, manifest in the out-of-plane anisotropy seen in $\mathrm{CoPt}$ and $\mathrm{Co} / \mathrm{Pd}$ multilayers [5-10]. Interlayer coupling can induce spatial frustration effects [5], domain wall pinning [8], and multidomain formation [7]. Further complexity occurs if perpendicular anisotropy structures are coupled to systems with in-plane anisotropy [11-13]. Understanding coupling between hard and soft regions is essential for the development of a wide range of technologies, including multilayer data storage media $[14,15]$, radio-frequency nano-oscillators $[16,17]$, and artificial multiferroics [18-20]. Recently, we identified a mode of vortex core polarity reversal in $\mathrm{Co} / \mathrm{Pd}-\mathrm{NiFe}$ multilayer systems, in which reversal occurs via the collapse of the core magnetization to a Bloch core with no out-of-plane component [13]. Crucially, this mode requires mutual spin imprinting between the layers [12], resulting in a complex superposition of a Landau state onto a maze domain pattern. Theoretical understanding of hybrid systems has been based on the approximation that the spin structure only varies across the layer boundary. While this approximation is valid for single-domain particles, it cannot describe the multidomain structures required to support Bloch core formation.

\section{MICROMAGNETIC HYBRID MODEL}

In this paper, we use micromagnetic and analytical modeling to investigate the novel hybrid Landau-maze domain phase in $\mathrm{Co} / \mathrm{Pd}-\mathrm{NiFe}$ multilayer systems, which is driven by a complex interaction between competing anisotropies. Despite the complexity, the essential characteristics of the three-dimensional (3D) magnetic structure are reproduced

*g.hrkac@exeter.ac.uk using analytical models. Combined with understanding of Bloch core formation, control over the domain phase could establish a new class of vortex oscillator, where core reversal is suppressed by confining oscillation within a multidomain structure. We use a finite element/boundary element method [21] to solve the Landau-Lifshitz-Gilbert equation for heterogeneous squares of width $w=800 \mathrm{~nm}$, consisting of a Permalloy $\left(\mathrm{Ni}_{80} \mathrm{Fe}_{20}\right.$, Py) layer of thickness $t_{\text {Py }}=10-50 \mathrm{~nm}$ coupled to a $\mathrm{Co} / \mathrm{Pd}$ multilayer of thickness $t_{\mathrm{Co} / \mathrm{Pd}}=9.6 \mathrm{~nm}$ [Fig. 1(a)] with an intergrain exchange model that calculates the exchange energy at the interface via a Mean-field approach [22]. Standard material parameters are used for Py (exchange stiffness $A_{\mathrm{Py}}=13 \mathrm{p} \mathrm{Jm}^{-1}$, saturation magnetization $M_{\mathrm{Py}}=800 \mathrm{kAm}^{-1}$, exchange length $l_{\mathrm{Py}}=5.7 \mathrm{~nm}$, magnetocrystalline anisotropy constant $K_{\mathrm{Py}}=0 \mathrm{~kJ} \mathrm{~m}^{-3}$, and damping $\left.\alpha_{\mathrm{Py}}=0.02\right)$. In general, the perpendicular anisotropy constant $K_{\mathrm{Co} / \mathrm{Pd}}$ and saturation magnetization $M_{\mathrm{Co} / \mathrm{Pd}}$ of the $\mathrm{Co} / \mathrm{Pd}$ multilayer depend on the thicknesses of the constituent layers [23]. Therefore, a range of $K_{\mathrm{Co} / \mathrm{Pd}}$ and $M_{\mathrm{Co} / \mathrm{Pd}}$ are used to establish the effect of the layer composition, but for simplicity, we assume the exchange constant and damping parameter are constant $\left(A_{\mathrm{Co} / \mathrm{Pd}}=10 \mathrm{pJ} \mathrm{m}^{-1}\right.$ and $\left.\alpha_{\mathrm{Co} / \mathrm{Pd}}=0.02\right)$. Variation in $M_{\mathrm{Co} / \mathrm{Pd}}$ produces a range of exchange lengths $l_{\mathrm{Co} / \mathrm{Pd}}=5-$ $40 \mathrm{~nm}$. We use an adaptive mesh increasing from $4 \mathrm{~nm}$ at the center of the square to $12 \mathrm{~nm}$ at the edges, enabling the smallest magnetic features in the vortex core and surrounding domain walls to be resolved while optimizing the computation time.

Individual $\mathrm{Py}$ and $\mathrm{Co} / \mathrm{Pd}$ layers exhibit different magnetic structures, determined by the dominant anisotropy. Shape anisotropy dominates the Py layer, causing the spins to form a flux-closing Landau state: four in-plane triangular $90^{\circ}$ domains with a central out-of-plane vortex core [Fig. 1(b)]. In the $\mathrm{Co} / \mathrm{Pd}$ layer, the perpendicular magnetic anisotropy dominates, resulting in a single-domain configuration with polar spin angle $\theta=0$ radians [Fig. 1(c)]. When the layers are in contact, exchange coupling causes imprinting of the spin state from one layer into the other. The coupling causes the in-plane magnetic state in both layers to be dominated by shape anisotropy, most often a Landau state, but more complex demagnetizing patterns emerge depending on $K_{\mathrm{Co} / \mathrm{Pd}}$ and $M_{\mathrm{Co} / \mathrm{Pd}}$. The imprinting of the in-plane state in the $\mathrm{Co} / \mathrm{Pd}$ layer arises because the exchange 
(a)

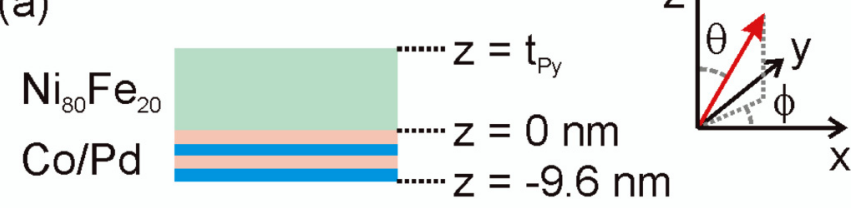

(b)
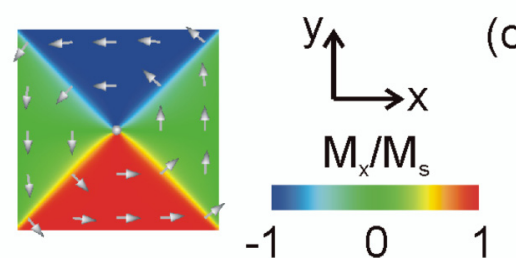

(c)

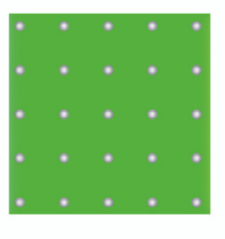

FIG. 1. (a) Schematic of the $\mathrm{Ni}_{80} \mathrm{Fe}_{20} / \mathrm{Co} / \mathrm{Pd}$ structure. Micromagnetic calculations of the individual layers show (b) Landau pattern formation in Permalloy and (c) $\mathrm{Co} / \mathrm{Pd}$ adopts out-of-plane spin structure $\left(t_{\mathrm{Py}}=30 \mathrm{~nm}, M_{\mathrm{Co} / \mathrm{Pd}}=400 \mathrm{kA} \mathrm{m}^{-1}\right.$, and $K_{\mathrm{Co} / \mathrm{Pd}}=$ $150 \mathrm{~kJ} \mathrm{~m}^{-3}$, arrows indicate the magnetization vector).

coupling across the interface requires the spin orientation to be continuous. Analogous to the magnetization rotation through a domain wall, the spin orientation cannot change abruptly when the local anisotropy direction changes across the boundary from out of plane $(\mathrm{Co} / \mathrm{Pd})$ to in plane $(\mathrm{Py})$, but rotates from one anisotropy direction to another over a characteristic length scale.

Competition between the perpendicular anisotropy and the shape anisotropy (varied here via $M_{\mathrm{Co} / \mathrm{Pd}}$ and $t_{\mathrm{Py}}$ ) leads to the generation of three distinctive magnetic phases that can be characterized according to the out-of-plane spin structure in the $\mathrm{Co} / \mathrm{Pd}$ layer. In the planar phase [Fig. 2(a)], the combined effect of the shape anisotropy and the frustration imposed by the Py layer suppresses out-of-plane magnetization in both Py and $\mathrm{Co} / \mathrm{Pd}$ layers, so the spin orientation throughout the structure is similar to that of the isolated Py layer [Fig. 1(b)]. The perpendicular phase [Fig. 2(a)] occurs when the perpendicular anisotropy overcomes the shape anisotropy, allowing the spins in the $\mathrm{Co} / \mathrm{Pd}$ layer to relax to the out-of-plane direction. When neither anisotropy is dominant, the structure enters the multidomain phase, characterized by the spins in the $\mathrm{Co} / \mathrm{Pd}$ layer buckling to form an out-of-plane maze domain pattern [Fig. 2(a)], so that the polar spin angle $\theta$ varies laterally, as well as through the structure thickness. Maze domains are a type of flux closure pattern with higher dipolar energy than an in-plane Landau pattern, as the flux closure is less efficient. In the multidomain phase, the dipolar energy cost of adopting the maze domain configuration is compensated by the perpendicular anisotropy energy saved through canting spins out of plane, so that the overall energy is minimized.

\section{ANALYTICAL PHASE BOUNDARY MODEL}

To probe the origins of the phase transitions, we derive a one-dimensional (1D) analytical model that assesses the energy benefit of forming a domain wall. It is based on the approximations that the planar and perpendicular phases are uniformly magnetized in and out of plane, respectively; the multidomain phase is treated as an out-of-plane two-domain state, and the energy balance in the $\mathrm{Co} / \mathrm{Pd}$ layer determines the (a)

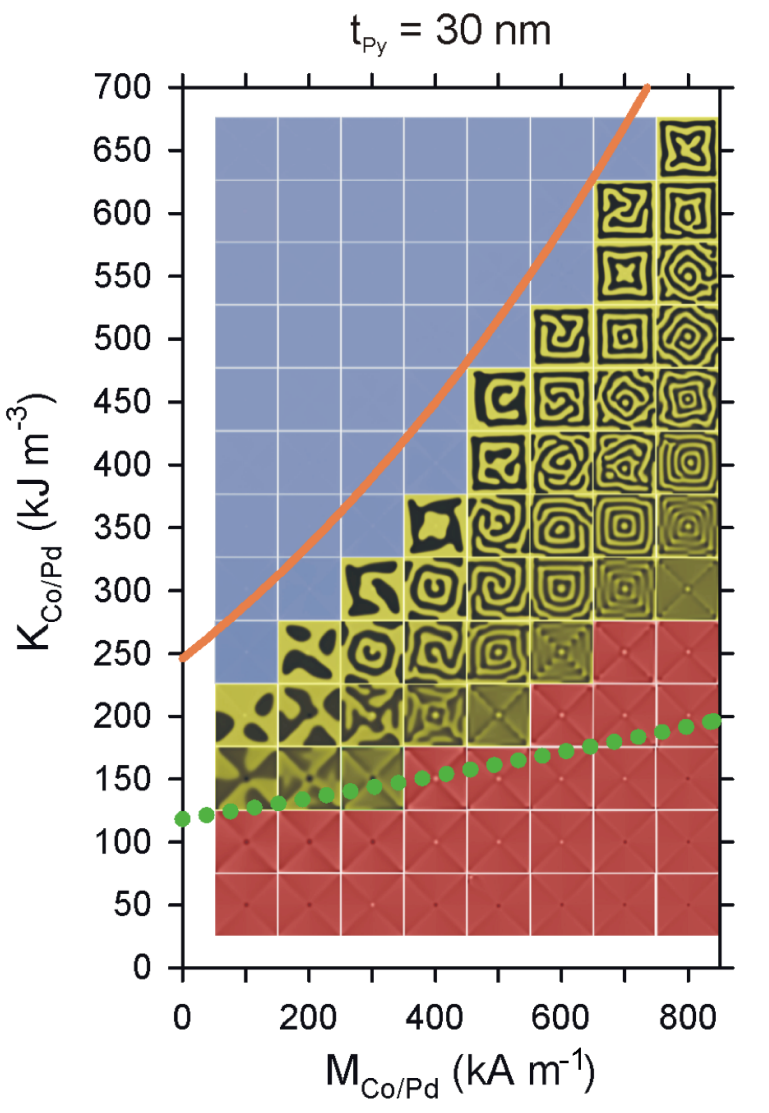

(b) $\quad t_{P y}=10 n m \quad t_{P y}=20 n m \quad t_{P y}=50 n m$

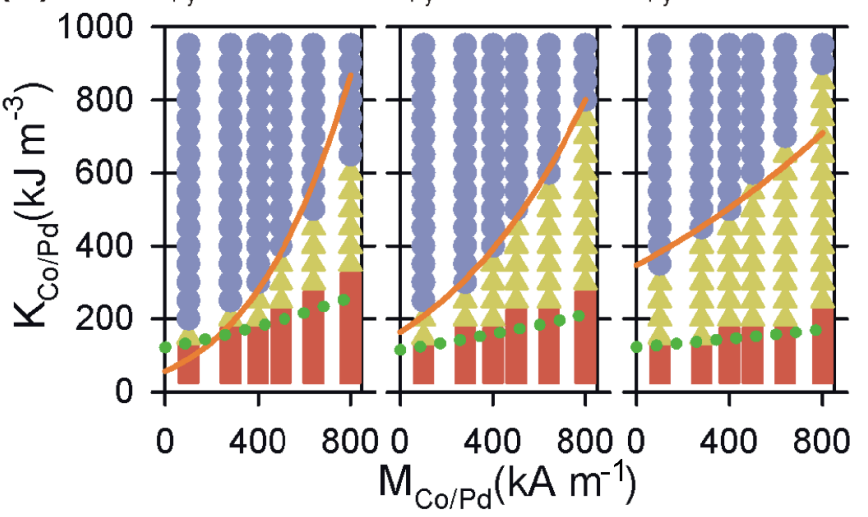

\begin{tabular}{|llll}
\hline - & Perpendicular phase & & \\
A & Multi-domain phase & 1D upper \\
- Planar phase & $\cdots$ & 1D lower \\
\hline
\end{tabular}

FIG. 2. (a) Domain phase diagram for $t_{\mathrm{Py}}=30 \mathrm{~nm}$ structure show how $\mathrm{Co} / \mathrm{Pd}$ material properties affect the remanent out-of-plane domain state in the $\mathrm{Co} / \mathrm{Pd}$ layer $(z=-9.6 \mathrm{~nm})$. Grayscale shading has been colorized to indicate the designated phase according to the color scheme shown in the legend. The lines plot the 1D predictions of the upper and lower domain phase boundaries, given in Eqs. (3) and (5), respectively. (b) Effect of $\mathrm{Co} / \mathrm{Pd}$ material properties on the magnetic domain phase, $t_{\mathrm{Py}}=10,20$, and $50 \mathrm{~nm}$.

phase transitions of the whole structure. Nonuniform magnetization through the sample thickness is taken into account by introducing a phenomenological exchange energy term 
when calculating the domain energies of the perpendicular and mixed phases. We take into account the interaction with the Py layer by defining the effective shape anisotropy constants in the $x, y$, or $z$ directions $K_{\mathrm{eff}}^{x, y, z}$ for the whole structure using an average magnetization weighted by the layer thicknesses

$$
K_{\mathrm{eff}}^{x, y, z}=\frac{1}{2} \mu_{o}\left(\frac{M_{\mathrm{Co} / \mathrm{Pd}} t_{\mathrm{Co} / \mathrm{Pd}}+M_{\mathrm{Py}} t_{\mathrm{Py}}}{t}\right)^{2} N_{x, y, z},
$$

where the total structure thickness $t=t_{\mathrm{Co} / \mathrm{Pd}}+t_{\mathrm{Py}}$, the demagnetization factors along the $x$ and $y$ directions for a square are $N_{x}=N_{y}=\left(1-N_{z}\right) / 2$, and the demagnetization factor along the $z$ direction $N_{z}$ takes the form proposed by Porter and Donahue [24], $\quad N_{z}=1-\frac{2}{\pi} \arctan (\rho)+\frac{1}{2 \pi} \rho^{-1} \ln \left(1+\rho^{2}\right)-$ $\frac{1}{2 \pi} \rho \ln \left(1+\rho^{-2}\right)$ with $\rho=\left(t_{\mathrm{Co} / P d}+t_{\mathrm{Py}}\right) / w$. Under these approximations, the upper phase boundary (between the perpendicular and multidomain phases) is determined by the transition from an out-of-plane single-domain state to an out-of-plane two-domain state.

\section{A. Upper phase boundary model}

The single-to-two domain phase transition has previously been derived for spherical particles with uniaxial anisotropy by considering that the formation of the two-domain state halves the demagnetization energy, but at the additional energy cost of introducing a domain wall [25]. Hence, the particle must be single domain if the domain wall energy is larger than half the demagnetization energy

$$
\sigma\left(\pi r^{2}\right) \geqslant \frac{1}{2} K_{\mathrm{eff}}^{z}\left(\frac{4}{3} \pi r^{3}\right),
$$

where $r$ is the radius of the sphere and $\sigma$ is the domain wall surface energy. For the given material parameters, Eq. (2) can be rearranged to find the critical radius $r_{c}=3 \sigma /\left(2 K_{\text {eff }}^{z}\right)$ below which the particle is single domain. Extending this solution to nonspherical structures, $r_{c}$ is interpreted as defining the critical domain wall surface area $\left(\pi r_{c}^{2}\right)$ required for domain wall formation. In the hybrid structure considered here, there is no perpendicular anisotropy in the Py layer, so the domain wall energy is concentrated in the $\mathrm{Co} / \mathrm{Pd}$ layer and $\sigma=4 \sqrt{A_{\mathrm{Co} / \mathrm{Pd}}\left(K_{\mathrm{Co} / \mathrm{Pd}}+K_{\text {eff }}^{x}\right)}$. Hence, the structure will be single domain if the critical domain wall surface area is larger than the cross-sectional area of the $\mathrm{Co} / \mathrm{Pd}$ layer $\pi r_{c}^{2} \geqslant w t_{\mathrm{Co} / \mathrm{Pd}}$. Therefore, the upper domain phase boundary between the perpendicular and multidomain states $\left.K_{\mathrm{Co} / \mathrm{Pd}}\right|_{\text {upper }}$ can be expressed as

$$
\left.K_{\mathrm{Co} / \mathrm{Pd}}\right|_{\text {upper }}=\frac{w t_{\mathrm{Co} / \mathrm{Pd}}}{36 \pi A_{\mathrm{Co} / \mathrm{Pd}}}\left(K_{\mathrm{eff}}^{z}\right)^{2}-K_{\mathrm{eff}}^{x} .
$$

\section{B. Lower phase boundary model}

The lower phase boundary (between the multidomain and planar phases) is determined by the balance of energies between the uniformly in-plane magnetized state and the out-of-plane two-domain state. The $\mathrm{Co} / \mathrm{Pd}$ layer will be magnetized in plane if the energy required for spins to lie along the hard axis of the perpendicular anisotropy is less than the energy to form a two-domain state with spins canted out of plane. Therefore, unlike in the upper domain phase, the spin orientation of the domains dominates the energy difference between the planar and multidomain phases, so the domain wall energy may be neglected. In the planar domain phase, the magnetization is uniformly in plane, so the total energy $E_{\text {planar }}$ is given by $E_{\text {planar }}=\left(K_{\mathrm{Co} / \mathrm{Pd}}+K_{\text {eff }}^{x}\right) w^{2} t_{\mathrm{Co} / \mathrm{Pd}}$.

The energy of the multidomain $E_{\text {multi }}$ state is determined by the dipolar energy and the exchange energy $E_{\mathrm{ex}}$ associated with the spin imprinting $E_{\text {multi }}=K_{\text {eff }}^{z} w^{2} t_{\mathrm{Co} / \mathrm{Pd}} / 2+E_{\mathrm{ex}}$. To evaluate $E_{\mathrm{ex}}$, we find the average exchange constant $A_{\mathrm{avg}}$ using an average weighted by the layer thickness $A_{\text {avg }}=$ $\left(A_{\mathrm{Co} / \mathrm{Pd}} t_{\mathrm{Co} / \mathrm{Pd}}+A_{\mathrm{Py}} t_{\mathrm{Py}}\right) / t$ and apply an approximation that the polar angle varies linearly by $\pi / 2\left(90^{\circ}\right)$ through the structure thickness $(\Delta \theta / \Delta z=(\pi / 2) / t)$

$$
E_{\mathrm{ex}}=A_{\mathrm{avg}}\left(\frac{\pi}{2 t}\right)^{2} w^{2} t_{\mathrm{Co} / \mathrm{Pd}}
$$

The planar domain phase occurs when $E_{\text {planar }} \leqslant E_{\text {multi }}$, so the lower domain phase boundary $\left.K_{\mathrm{Co} / \mathrm{Pd}}\right|_{\text {lower }}$ is

$$
\left.K_{\mathrm{Co} / \mathrm{Pd}}\right|_{\text {lower }}=\frac{1}{2} K_{\mathrm{eff}}^{z}-K_{\mathrm{eff}}^{x}+A_{\mathrm{avg}}\left(\frac{\pi}{2 t}\right)^{2} .
$$

\section{Spin structure across interface}

Despite the simplicity of the approximations used, the phase boundaries predicted by Eqs. (3) and (5) are in reasonable agreement with the micromagnetic model (Fig. 2), given that there are no fitted parameters. Divergence between the two models is greatest at the extremes of $M_{\mathrm{Co} / \mathrm{Pd}}$. For the 10 nm thick Py layer, the 1D model predicts that the upper domain phase boundary falls below the lower domain phase boundary at low $M_{\mathrm{Co} / \mathrm{Pd}}$, causing the multidomain phase to vanish [Fig. 2(b)]. The micromagnetic model shows that the multidomain phase in fact persists even at the lowest $M_{\mathrm{Co} / \mathrm{Pd}}$, albeit over a very narrow range of $K_{\mathrm{Co} / \mathrm{Pd}}$. At the other extreme, the $1 \mathrm{D}$ prediction for the highest $M_{\mathrm{Co} / \mathrm{Pd}}$ in the $t_{\mathrm{Py}}=10$ and $50 \mathrm{~nm}$ structures deviates from the micromagnetic model, due to a level of complexity that cannot be easily included in the 1D model. These effects include nonuniform dipolar fields, which occur due to the structure shape [26] and heterogeneous composition, and the 2D magnetic structure of the Landau pattern, which enables energy dissipation mechanisms via modifications of the Landau domain wall width and structure. Experimentally, however, the compositions required to reach the extremes of $M_{\mathrm{Co} / \mathrm{Pd}}$ result in perpendicular anisotropy reduced below the lower phase boundary [23], so the regions of largest disagreement between the 1D and micromagnetic models are least significant. Therefore, the 1D model provides physical insight into the origins of the phase boundaries within experimentally accessible parameters.

Analyzing the change in the spin orientation through the structure thickness showed that the polar spin angle imprinting was strongest in the multidomain phase rather than the perpendicular domain phase, which might have been expected. The imprinting was studied for $t_{\mathrm{Py}}=30 \mathrm{~nm}$ in structures with $M_{\mathrm{Co} / \mathrm{Pd}}=400 \mathrm{kA} \mathrm{m}^{-1}$, such that each structure had a fixed shape anisotropy. Under this condition, the multidomain phase occurs for perpendicular anisotropy in the range $200 \mathrm{~kJ} \mathrm{~m}^{-3} \leqslant K_{\mathrm{Co} / \mathrm{Pd}} \leqslant 350 \mathrm{~kJ} \mathrm{~m}^{-3}$. In both the multidomain and perpendicular domain phases, out-of-plane spin angle canting [Figs. 3(a)-3(c)] originating in the $\mathrm{Co} / \mathrm{Pd}$ layer is transferred to the Py layer [Fig. 3(b)] at the interlayer 

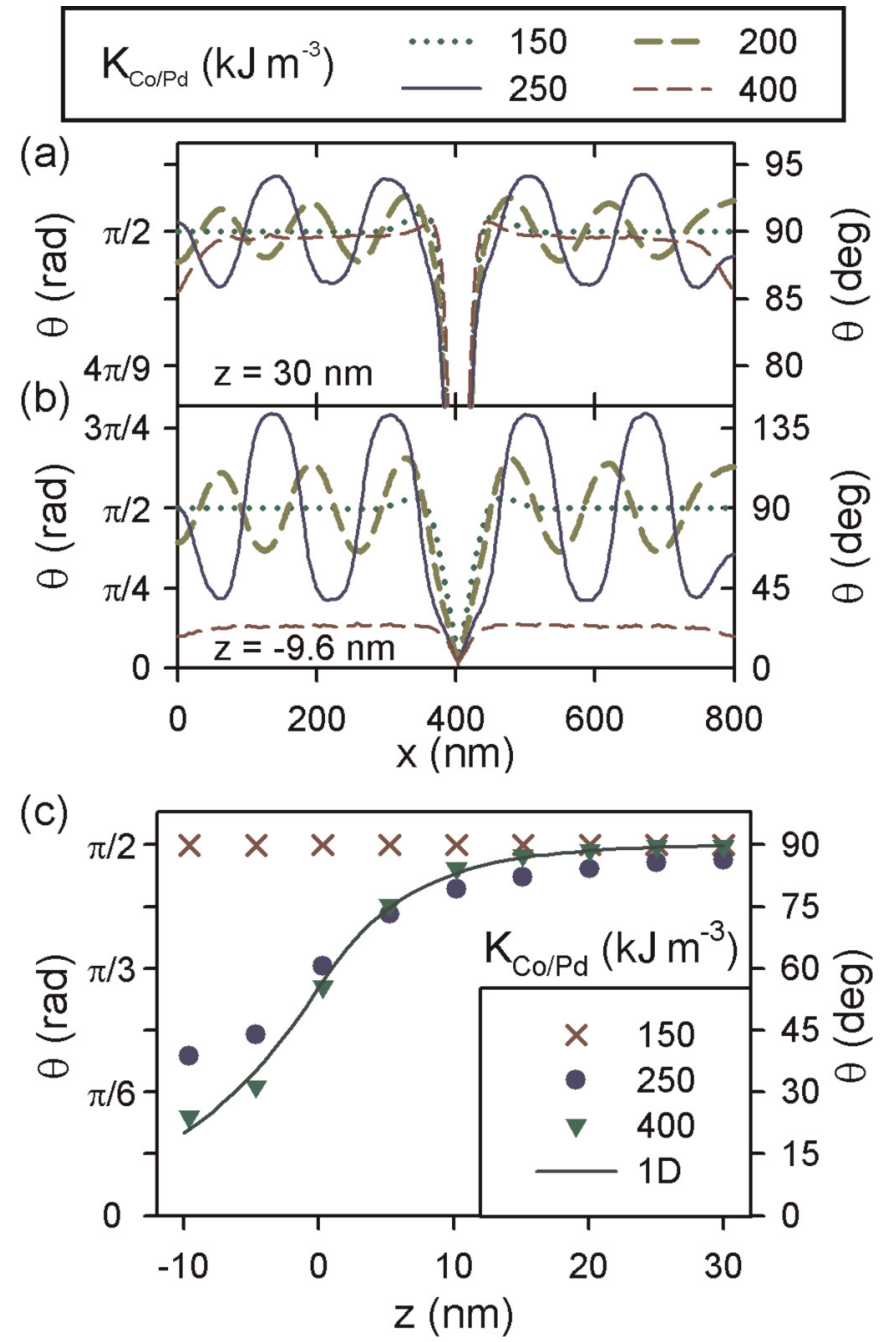

FIG. 3. Polar spin angle $\theta$ at various $K_{\mathrm{Co} / \mathrm{Pd}}$ along a line intersecting the vortex core (a) top surface of the Py layer and (b) bottom surface of the $\mathrm{Co} / \mathrm{Pd}$ layer. Vortex core at $x=400 \mathrm{~nm}$. Oscillations in $\theta$ are due to the profile intersecting different maze domains. (c) Variation of $\theta$ through the structure thickness. Bold line: $1 \mathrm{D}$ model prediction [Eqs. (6)-(8)] with $K_{\mathrm{Co} / \mathrm{Pd}}=400 \mathrm{~kJ} \mathrm{~m}^{-3}$. $t_{\mathrm{Py}}=30 \mathrm{~nm}$ and $M_{\mathrm{Co} / \mathrm{Pd}}=400 \mathrm{kA} \mathrm{m}^{-1}$ in all cases.

boundary, but steadily decays towards the in-plane state $\left(\theta=\pi / 2,90^{\circ}\right)$ through the Py thickness [Fig. 3(a)]. Within the multidomain phase, increases in $K_{\mathrm{Co} / \mathrm{Pd}}$ enhance the out-of-plane rotation and size of the maze domains [Fig. 3(b)], although the overall out-of-plane magnetization remains very low, as the contributions from opposing perpendicular maze domains cancel when integrating over the whole structure. In the perpendicular domain phase, no such cancellation occurs. However, on a local level, the polar spin angle at any point on the top surface of the Py layer is greater in the multidomain phase than the perpendicular domain phase [Fig. 3(a)]. Indeed, Fig. 3(c) shows that the polar spin angle decays less rapidly throughout the structure thickness when $K_{\mathrm{Co} / \mathrm{Pd}}=250 \mathrm{~kJ} \mathrm{~m}^{-3}$ than when $K_{\mathrm{Co} / \mathrm{Pd}}=400 \mathrm{~kJ} \mathrm{~m}^{-3}$. This means that the smaller perpendicular anisotropy imprints larger out-of-plane spin components into the Py layer.

To understand this counterintuitive behavior better, we considered models of the spin angle through the structure thickness. Initially, we evaluate a 1D analytical model, only considering $\theta$ variation along the $z$ axis $(\nabla \theta=d \theta / d z$, constant azimuth angle). The energy densities within the $\mathrm{Co} / \mathrm{Pd}\left(W_{\mathrm{Co} / \mathrm{Pd}}\right)$ and $\mathrm{Py}$ $\left(W_{\mathrm{Py}}\right)$ layers are given by $W_{\mathrm{Co} / \mathrm{Pd}}=A_{\mathrm{Co} / \mathrm{Pd}}\left(\left.\nabla \theta\right|_{\mathrm{Co} / \mathrm{Pd}}\right)^{2}+$ $\left(K_{\mathrm{Co} / \mathrm{Pd}}+K_{\mathrm{eff}}^{x}\right) \sin ^{2} \theta+K_{\mathrm{eff}}^{z} \cos ^{2} \theta$ and $W_{\mathrm{Py}}=A_{\mathrm{Py}}\left(\left.\nabla \theta\right|_{\mathrm{Py}}\right)^{2}+$ $K_{\mathrm{eff}}^{x} \sin ^{2} \theta+K_{\mathrm{eff}}^{z} \cos ^{2} \theta$. For comparison with the micromagnetic data in Fig. 3, the effective shape anisotropy constants calculated using Eq. (1) with $M_{\mathrm{Co} / \mathrm{Pd}}=400 \mathrm{kA} \mathrm{m}^{-1}$ are $K_{\text {eff }}^{\mathrm{x}}=$ $K_{\text {eff }}^{y}=10 \mathrm{~kJ} \mathrm{~m}^{-3}$ and $K_{\text {eff }}^{z}=290 \mathrm{~kJ} \mathrm{~m}^{-3}$. Within each layer, the gradient of the polar angle $\nabla \theta$ may be derived by minimization of the energy density with respect to the polar angle [18]

$$
\begin{gathered}
\left.\nabla \theta\right|_{\mathrm{Py}}=\sqrt{\frac{K_{\mathrm{eff}}^{z}-K_{\mathrm{eff}}^{x}}{\mathrm{~A}_{\mathrm{Py}}}} \cos \theta, \\
\left.\nabla \theta\right|_{\mathrm{Co} / \mathrm{Pd}}=\left\{\begin{array}{ll}
\sqrt{\frac{K_{\mathrm{Co} / \mathrm{Pd}}+K_{\mathrm{eff}}^{x}-K_{\mathrm{eff}}^{z}}{\mathrm{~A}_{\mathrm{Co} / \mathrm{Pd}}} \sin \theta,} & K_{\mathrm{Co} / \mathrm{Pd}}+K_{\mathrm{eff}}^{x}>K_{\mathrm{eff}}^{z} \\
\sqrt{\frac{K_{\mathrm{eff}}^{z}-K_{\mathrm{eff}}^{x}-K_{\mathrm{Co} / \mathrm{Pd}}}{\mathrm{A}_{\mathrm{Co} / \mathrm{Pd}}}} \cos \theta, & K_{\mathrm{Co} / \mathrm{Pd}}+K_{\mathrm{eff}}^{x} \leqslant K_{\mathrm{eff}}^{z}
\end{array} .\right.
\end{gathered}
$$

To understand the variation of $\theta$ through the structure thickness, we do not impose any boundary condition on the external surfaces. However, as the layers are strongly coupled, we apply the condition that the spin orientation varies continuously across the interlayer boundary, so $\left.\nabla \theta\right|_{\mathrm{Co} / \mathrm{Pd}}=$ $\left.\nabla \theta\right|_{\mathrm{Py}}$ at $z=0 \mathrm{~nm}$. Using this boundary condition, Eqs. (6) and (7) can be numerically integrated to define the polar angle profile along the $z$ direction. Equating Eq. (6) and (7), we find

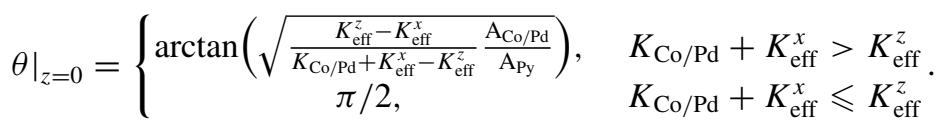

Figure 3(c) shows that Eqs. (6)-(8) produce good agreement with the micromagnetic model in the perpendicular $\left(K_{\mathrm{Co} / \mathrm{Pd}} \geqslant\left. K_{\mathrm{Co} / \mathrm{Pd}}\right|_{\text {upper }}\right)$ and planar $\left(K_{\mathrm{Co} / \mathrm{Pd}} \leqslant\left. K_{\mathrm{Co} / \mathrm{Pd}}\right|_{\text {lower }}\right)$ phases, where there is little lateral change in $\theta$. However, Eqs. (6)-(8) cannot describe the multidomain phase present in the micromagnetic model, as an analytical model of the maze domain imprinting would require at least a two-dimensional (2D) formulation of the polar angle gradient to describe variation of $\theta$ along both the $x$ and $z$ axes.

As $2 \mathrm{D}$ or $3 \mathrm{D}$ solutions to this system cannot be found by analytical methods, we qualitatively assess the impact that an additional degree of freedom along the $x$ axis has 
on each energy term. As the structure thickness is much smaller than the width, demagnetizing fields are uniform (neglecting edge effects), so the demagnetization energy is largely unaffected by the additional degree of freedom. The perpendicular anisotropy energy is similarly unchanged in a 2D system, since the anisotropy direction is constant. However, the exchange energy is dependent on the polar angle gradient, so is the only term altered by variation of the polar angle along both the $x$ and $z$ axes (for a 2D system, $\nabla \theta=\partial \theta / \partial x+\partial \theta / \partial z$ ). The presence of domain walls reduces the average change in $\theta$ through the structure thickness (compared to the 1D case), since at the wall center $\theta=\pi / 2$ for all $z$. Therefore, unlike the 1D analysis presented in Eqs. (6) and (7), in the center of the domain wall $\partial \theta / \partial z=0$. Compensating this reduction in $\partial \theta / \partial z$ is $\partial \theta / \partial x$, the lateral change in $\theta$, which is maximal at the wall center and minimal within domains. In essence, domain texture enables the exchange energy to dissipate over two dimensions rather than one, reducing the total rotation of $\theta$ through the sample thickness compared with 1D systems.

\section{CONCLUSIONS}

In conclusion, we have characterized a multidomain phase that enhances mutual spin imprinting from a hard $\mathrm{Co} / \mathrm{Pd}$ layer into a soft Py layer. Formation of the multidomain phase is dependent on the shape anisotropy of the structure and the perpendicular anisotropy of the Co/Pd layer, both of which can be tailored experimentally. Thus, we describe how the hybrid Landau-maze domain state could be selected experimentally in order to explore its potential functionality, for example, in magnetic oscillators based on vortex core precession $[16,17]$.

\section{ACKNOWLEDGMENTS}

The authors acknowledge support from the Vienna Science and Technology Fund (WWTF) under Grant No. MA14-44, the Engineering and Physical Sciences Research Council (EPSRC) Grant No. EP/K008412/1, and from the Royal Society under Grant No. UF080837.
[1] L. J. Heyderman and R. L. Stamps, J. Phys.: Condens. Matter 25, 363201 (2013).

[2] W. H. Meiklejohn and C. P. Bean, Phys. Rev. 102, 1413 (1956).

[3] P. Blomqvist, K. M. Krishnan, and H. Ohldag, Phys. Rev. Lett. 94, 107203 (2005).

[4] J. Camarero, J. Sort, A. Hoffmann, J. M. Garcia-Martin, B. Dieny, R. Miranda, and J. Nogues, Phys. Rev. Lett. 95, 057204 (2005).

[5] J. Briones, F. Montaigne, D. Lacour, M. Hehn, M. J. Carey, and J. R. Childress, Appl. Phys. Lett. 92, 032508 (2008).

[6] N. Rougemaille, F. Montaigne, B. Canals, A. Duluard, D. Lacour, M. Hehn, R. Belkhou, O. Fruchart, S. El Moussaoui, A. Bendounan, and F. Maccherozzi, Phys. Rev. Lett. 106, 057209 (2011).

[7] S. Mangin, T. Hauet, P. Fischer, D. H. Kim, J. B. Kortright, K. Chesnel, E. Arenholz, and E. E. Fullerton, Phys. Rev. B 78, 024424 (2008).

[8] P. J. Metaxas, P.-J. Zermatten, J.-P. Jamet, J. Ferre, G. Gaudin, B. Rodmacq, A. Schuhl, and R. L. Stamps, Appl. Phys. Lett. 94, 132504 (2009).

[9] A. Berger, D. T. Margulies, and H. Do, Appl. Phys. Lett. 85, 1571 (2004).

[10] A. Berger, O. Hovorka, G. Friedman, and E. E. Fullerton, Phys. Rev. B 78, 224407 (2008).

[11] G. Asti, M. Ghidini, R. Pellicelli, C. Pernechele, M. Solzi, F. Albertini, F. Casoli, S. Fabbrici, and L. Pareti, Phys. Rev. B 73, 094406 (2006).

[12] G. Heldt, M. T. Bryan, G. Hrkac, S. E. Stevenson, R. V. Chopdekar, J. Raabe, T. Thomson, and L. J. Heyderman, Appl. Phys. Lett. 104, 182401 (2014).

[13] P. Wohlhüter, M. T. Bryan, P. Warnicke, S. Gliga, S. E. Stevenson, G. Heldt, L. Saharan, A. K. Suszka, C. Moutafis,
R. V. Chopdekar, J. Raabe, T. Thomson, G. Hrkac, and L. J. Heyderman, Nature Commun. 6, 7836 (2015).

[14] O. Hellwig, A. Berger, T. Thomson, E. Dobisz, Z. Z. Bandic, H. Yang, D. S. Kercher, and E. E. Fullerton, Appl. Phys. Lett. 90, 162516 (2007).

[15] M. Albrecht, G. Hu, A. Moser, O. Hellwig, and B. D. Terris, J. Appl. Phys. 97, 103910 (2005).

[16] Q. Mistral, M. van Kampen, G. Hrkac, J.-V. Kim, T. Devolder, P. Crozat, C. Chappert, L. Lagae, and T. Schrefl, Phys. Rev. Lett. 100, 257201 (2008).

[17] I. Tudosa, J. A. Katine, S. Mangin, and E. E. Fullerton, Appl. Phys. Lett. 96, 212504 (2010).

[18] M. T. Bryan, J. Dean, and D. A. Allwood, Phys. Rev. B 85, 144411 (2012).

[19] U. Bauer, M. Przybylski, J. Kirschner, and G. S. D. Beach, Nano Lett. 12, 1437 (2012).

[20] N. Lei, T. Devolder, G. Agnus, P. Aubert, L. Daniel, J.-V. Kim, W. Zhao, T. Trypiniotis, R. P. Cowburn, C. Chappert, D. Ravelosona, and P. Lecoeur, Nature Commun. 4, 1378 (2013).

[21] D. Suess, V. Tsiantos, T. Schrefl, J. Fidler, W. Scholz, H. Forster, R. Dittrich, and J. J. Miles, J. Magn. Magn. Mater. 248, 298 (2002).

[22] D. Suess, J. Magn. Magn. Mater. 308, 183 (2007).

[23] O. Hellwig, T. Hauet, T. Thomson, E. Dobisz, J. D. RisnerJamtgaard, D. Yaney, B. D. Terris, and E. E. Fullerton, Appl. Phys. Lett. 95, 232505 (2009).

[24] D. G. Porter and M. J. Donahue, J. Appl. Phys. 95, 6729 (2004).

[25] S. Chikazumi, Physics of Ferromagnetism, 2nd ed. (Oxford University Press, New York, 1997).

[26] X. F. Han, M. Grimsditch, J. Meersschaut, A. Hoffmann, Y. Ji, J. Sort, J. Nogues, R. Divan, J. E. Pearson, and D. J. Keavney, Phys. Rev. Lett. 98, 147202 (2007). 